\title{
Diffuse Cloud Models: Successes and Challenges
}

\author{
E. Roueff, M. Ruaud, F. Le Petit, B. Godard and J. Le Bourlot
}

LUTh and UMR 8102, Observatoire de Paris, F 92190 Meudon, France

email: evelyne.roueff@obspm.fr

\begin{abstract}
We present the general physical conditions thought to be present in diffuse interstellar clouds. The radiation field is driving the atomic to molecular transition and the resulting physical conditions. We focus on the recent observational signatures of significant values of the cosmic ionization rate $\left(10^{-16}-10^{-15} \mathrm{~s}^{-1}\right)$ which should also impact the clues to Diffuse interstellar Bands.
\end{abstract}

Keywords. molecules, cosmic ionization, interstellar physics.

\section{Introduction}

Diffuse interstellar bands (DIBs) are detected in so-called diffuse or translucent environments which are definitely disconnected from stellar atmospheres. These interstellar locations are studied for themselves via different other spectral signatures which allow to derive their physical properties. Understanding and constraining the physical conditions is the main goal of diffuse cloud modeling, which also may help to finally resolve the mystery of the DIB carriers. Diffuse clouds are observed in all wavelength ranges, from the far vacuum ultraviolet up to millimeter wavelength and involve mainly absorption spectra as the environment is generally too "diffuse" to allow for excitation and subsequent emission. The continuum background is provided by hot bright stars in the UV and visible part of the spectrum whereas high mass star forming regions and even extragalactic quasars provide infrared, submillimeter and millimeter background located randomly. A variety of new instrumentation has allowed to significantly improve the observational knowledge and provide new insights on these diffuse interstellar regions. The VUV range gives access to the detection of both atomic and molecular hydrogen via their Lyman and Werner band systems and FUSE, the Far Ultraviolet Spectroscopic Explorer spatial instrument, has allowed to significantly expand the previous knowledge provided by the Copernicus satellite (Rachford et al. 2009). The visible / infrared spectrum can now be observed with great sensitivity and spectral resolution with $8 \mathrm{~m}$ class telescopes such as the VLT and the Keck telescopes and heterodyne technology in the submillimeter and millimeter also allows to provide a better insight of the chemical complexity of these diffuse environments.

We present in this review the general features of interstellar cloud models where the atomic to molecular transition is driven by the ultra-violet interstellar radiation field (ISRF). A general consensus has emerged on the basic physical conditions of diffuse interstellar clouds in the galactic plane which are recalled in Table 1.

Density and temperatures are derived from the rotational populations of molecular hydrogen (Rachford et al. 2009). Jenkins \& Tripp (2011) have constrained the values of thermal pressure in diffuse clouds through the analysis of fine structure populations of atomic carbon. These values are consistent with the temperatures and densities derived independently. The radiation field scaling factor $\chi$ is usually defined according to the 
Table 1. Overview of current physical conditions in diffuse clouds.

\begin{tabular}{|c|c|c|c|c|c|c|}
\hline $\begin{array}{c}\mathbf{n}_{H} \\
{\left[\mathbf{c m}^{-3}\right]}\end{array}$ & $\begin{array}{c}\mathbf{T} \\
{[\mathbf{K}]}\end{array}$ & $\left.\begin{array}{c}\log _{10} \mathbf{p} \\
\mathbf{p} \text { in }\left[\left(\mathbf{c m}^{-3}\right.\right.\end{array} \mathbf{K}\right]$ & $\begin{array}{c}\text { radiation field } \\
\text { scaling factor }\end{array}$ & $\begin{array}{c}\text { electronic } \\
\text { fraction }\end{array}$ & $\begin{array}{c}\text { molecular } \\
\text { fraction }\end{array}$ & $\begin{array}{c}\text { cosmic ionization } \\
\text { rate per } \mathbf{H}_{2} \zeta\left[\mathbf{s}^{-1}\right]\end{array}$ \\
\hline$\sim 50-100$ & $67 \pm 14^{(1)}$ & $3.6-4.3^{(2)}$ & $\chi \sim 1-10$ & $\mathrm{n}(\mathrm{e})=\mathrm{n}\left(\mathrm{C}^{+}\right)$ & $\mathrm{f} \sim 0.01-0.7$ & $5 \times 10^{-17}-5 \times 10^{-16}$ \\
\hline
\end{tabular}

Notes: ${ }^{1}$ Rachford et al. (2009). ${ }^{2}$ Jenkins \& Tripp (2011).

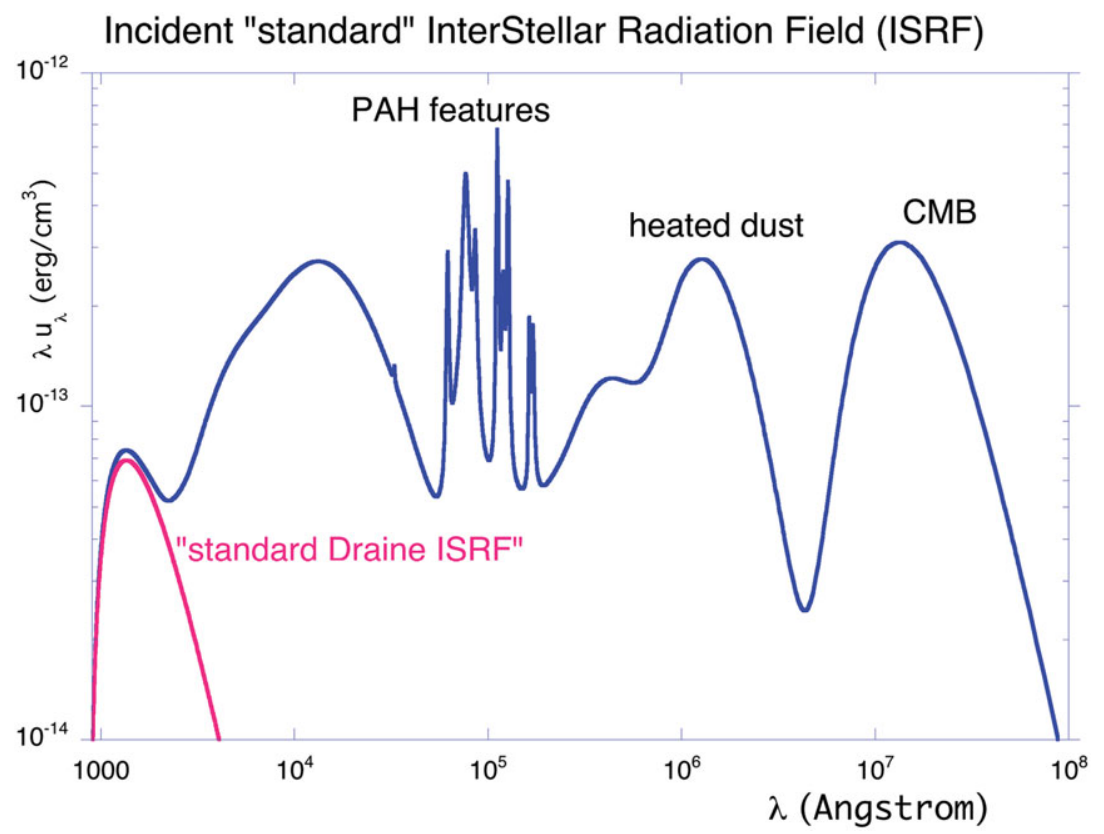

Figure 1. Standard ISRF model.

standard value of the InterStellar Radiation Field (ISRF) provided by Draine (1978). However, this scaling factor is essentially concerning the VUV part of the spectrum, i.e. from $13.6 \mathrm{eV}$ to $6 \mathrm{eV}$, which is the determining range for photodissociation effects. At longer wavelengths, signatures of PAH like molecules and heated dust are taking place and the cosmic microwave background extends up to millimeter wavelengths. Fig. 1 displays the corresponding spectrum as introduced in present PDR models (Le Petit et al. 2006, 2009; Le Bourlot et al. 2012).

The cutoff at short wavelengths is given by the ionization energy of atomic hydrogen, at $13.6 \mathrm{eV}$ corresponding to 912 Angstroms. Carbon, sulfur ions are photoionized at the edge of the cloud and the electronic fraction is often assumed to be equal to the fractional abundance of ionic carbon but this approximation may be revised as will be discussed later. The molecular fraction $f$ is defined as $f=\frac{2 \times N\left(H_{2}\right)}{N(H)+2 \times N\left(H_{2}\right)}$, where $\mathrm{N}(\mathrm{H})$ and $\mathrm{N}\left(\mathrm{H}_{2}\right)$ are the atomic (respectively molecular) observed column densities in the diffuse cloud, and ranges from very few to about 0.8 . Finally, the cosmic ionization rate is an additional parameter which has not been paid much attention until recently, as it was thought to play a minor role in diffuse cloud conditions where the main ionizing agent is the radiation field. We will discuss this particular point in section 3 . 


\section{Diffuse cloud models}

Diffuse clouds are a subclass of Photon Dominated Regions or PDRs, where densities and radiation fields display larger values than in diffuse clouds, allowing them to be detected in emission but the basic physical processes are similar and controlled by the radiation field. The atomic to molecular transition results from the balance between $\mathrm{H}_{2}$ formation on dust grain surfaces and photodissociation which takes place via discrete absorption transitions followed by fluorescence in the continuum of the $\mathrm{H}_{2}$ ground electronic state (Abgrall et al. 1992). PDR modeling is performed in different groups and a benchmark exercise has taken place in the Lorentz Center about 10 years ago, resulting in finally concordant results for simple well defined cases, as described in Röllig et al. (2007) for a dozen different groups. Differences between the various codes range from geometry assumptions, plane parallel or spherical "cloud", perpendicular or isotropic incident radiation field to radiative transfer treatments, implementation of dust effects and possible time dependence and the final agreement is remarkable.

We will, in the following, present results coming from the Meudon PDR code (Le Petit et al. 2002, 2006; Goicoechea \& Le Bourlot 2007; Gonzalez Garcia et al. 2008; Le Petit et al. 2009; Le Bourlot et al. 2012), developed in our team since more than 15 years, which is available on line at http://pdr.obspm.fr and used by different groups in Europe and USA. This code is updated regularly by including new physical mechanisms and allows to compute, at various levels of approximations, the chemical and thermal balance of the atomic to molecular transition of an infinite plane parallel slab of gas and dust. We display in Figure 2 the atomic to $\mathrm{H}$ to $\mathrm{H}_{2}$ and $\mathrm{C}^{+} / \mathrm{C} / \mathrm{CO}$ transitions for an isobaric diffuse cloud model which occur respectively at $\mathrm{A}_{V}=0.1$ and $\sim 2$.

\section{1. diffuse cloud chemistry}

Reactions between $\mathrm{H}_{2}$ and available atoms or atomic ions, depending on the ionization energies, are the first chemical steps in diffuse clouds. Figure 4 displays the energy released in the dissociation of the $\mathrm{XH}^{+}$hydride ion into $\mathrm{X}+\mathrm{H}^{+}$in function of the dissociation energy of the neutral hydride $\mathrm{XH}$, the energy released into $\mathrm{X}+\mathrm{H}$. Atoms with ionization potentials lower than $13.6 \mathrm{eV}$ are displayed as red diamonds. The vertical (horizontal) dashed line corresponds to the dissociation energy of $\mathrm{H}_{2}$.

This figure, inspired by that reported in Neufeld \& Wolfire (2009), allows to directly infer which reactions are likely to proceed. For atomic ions, only $\mathrm{Cl}^{+}, \mathrm{O}^{+}$and $\mathrm{F}^{+}$, which are located above the horizontal dashed line, will react exothermically with $\mathrm{H}_{2}$ and produce $\mathrm{XH}^{+}$. Then, we see that $\mathrm{Cl}^{+}$is the only ion which is photoionized and may react directly with $\mathrm{H}_{2}$ to form $\mathrm{HCl}^{+}$, which has been detected recently by De Luca et al. (2012). $\mathrm{O}^{+}$and $\mathrm{F}^{+}$, may also react with $\mathrm{H}_{2}$ but these ions can only be produced through cosmic ray or $\mathrm{X}$ ray ionizations. Other diatomic hydride molecular ions built from photoionized atoms, including $\mathrm{CH}^{+}$, which is one of the first interstellar molecular species discovered, require some energy input in order to overcome the endothermicity of the $X^{+}+\mathrm{H}_{2} \rightarrow X H^{+}+H$ reaction. We may also derive from this figure the likely exothermic reactions involving neutral atoms and $\mathrm{H}_{2}$, which are found for species which lie to the right of the vertical dashed line. A single atom satisfies the constrain, i.e. F, which gives hydrogen fluorine in the $\mathrm{F}+\mathrm{H}_{2} \rightarrow \mathrm{HF}+\mathrm{H}$ exothermic and well theoretically studied reaction. Note however that a small barrier is present, and the behavior of the reaction rate at low temperatures depends strongly on non-adiabatic effects as discussed in Lique et al. (2011). Subsequent reactions occur between abundant ions such as $\mathrm{C}^{+}$, $\mathrm{S}^{+}$and hydrides $\mathrm{OH}, \mathrm{CH}, \ldots$ forming finally $\mathrm{CO}, \mathrm{C}_{2}, \mathrm{C}_{2} \mathrm{H}, \mathrm{C}_{3}$ etc...which are indeed present in diffuse molecular clouds (Snow \& McCall 2006). Whereas the presence of 


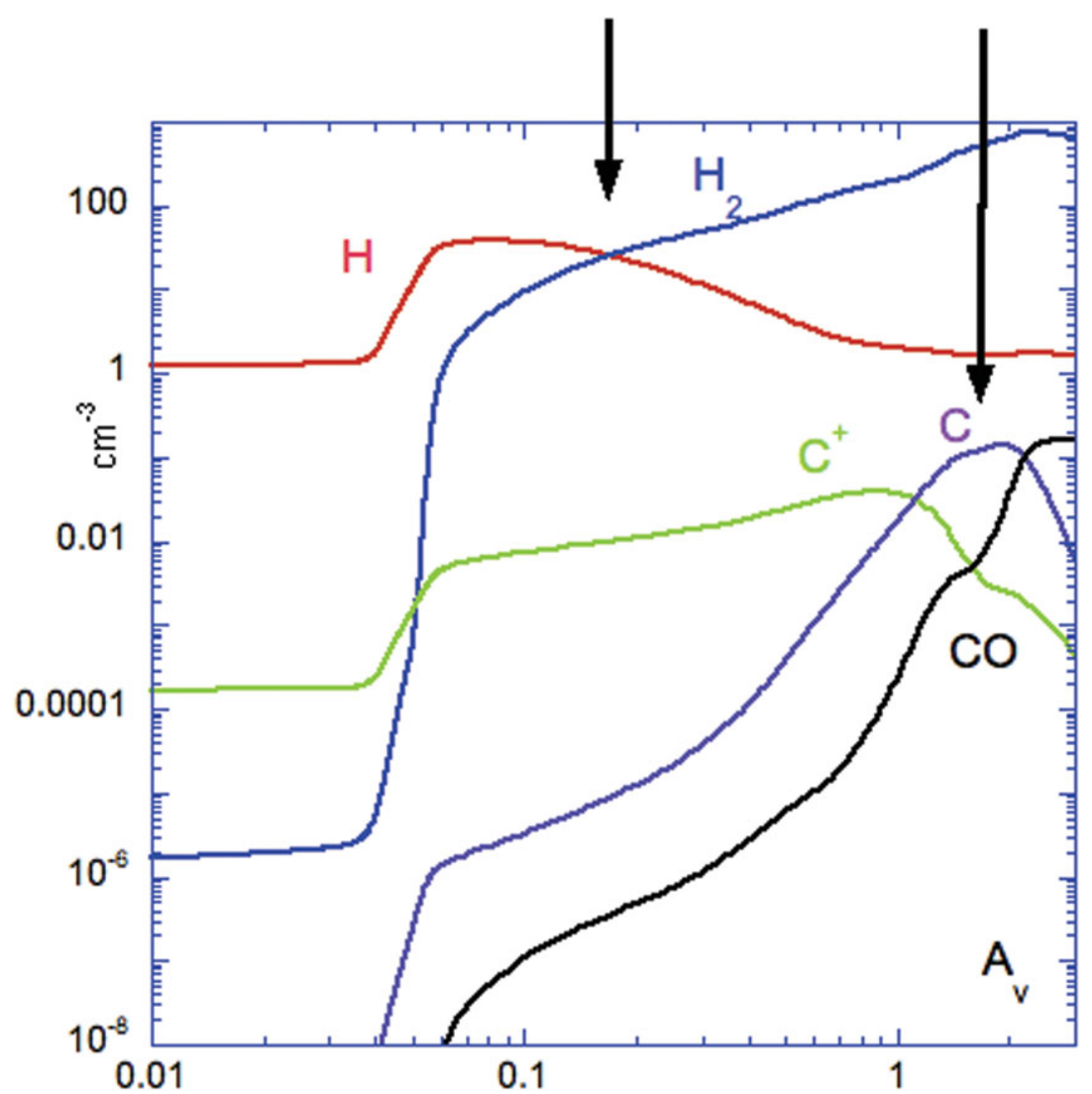

Figure 2. isobaric model $\log _{10} \mathrm{p}=3.9$. Volumic densities are displayed as a function of the visual extinction $A_{V} \cdot \chi=5, \zeta=10^{-16}$. Vertical arrows show the $\mathrm{H}$ to $\mathrm{H}_{2}$ and $\mathrm{C}^{+} / \mathrm{C} / \mathrm{CO}$ transitions.

some observed molecular ions $\left(\mathrm{CH}^{+}, \mathrm{SH}^{+}\right)$requires specific energy input, such as shocks or turbulence for the reasons explained above, most neutral molecules are released in dissociative recombination reactions of polyatomic molecular ions. The recent detection of the series $\mathrm{OH}^{+}, \mathrm{H}_{2} \mathrm{O}^{+}, \mathrm{H}_{3} \mathrm{O}^{+}$in various diffuse clouds (Neufeld et al. 2010) is a nice confirmation of the validity of the ion-molecule reaction schema introduced almost thirty years ago in van Dishoeck \& Black (1986).

\section{3. $\mathbf{H}_{3}^{+}$, a tracer of cosmic rays}

Values of the cosmic ray ionization can be derived from analyses of the interstellar chemical composition. It has been recognized since many years that $\mathrm{OH}, \mathrm{HD}$ are useful proxies of the cosmic ionization rate as the starting step of the chemistry involves charge transfer reactions with protons, coming from the cosmic ray ionization of atomic/molecular hydrogen. Values of several $10^{-17} \mathrm{~s}^{-1}$ were then derived (van Dishoeck \& Black 1986). This low value has been debated recently from two different point of views. Considering the possible loss of protons in collisions with grains, Liszt (2003) deduced a lower limit of the cosmic ionization rate of $2 \times 10^{-16} \mathrm{~s}^{-1}$ in order to explain the amount of $\mathrm{OH}$ and $\mathrm{HD}$ in diffuse clouds. The outstanding discovery of $\mathrm{H}_{3}^{+}$in diffuse clouds by McCall et al. $(2002,2003)$ has provided an additional even more direct probe of this important parameter. Formation/destruction balance of $\mathrm{H}_{3}^{+}$is directly resulting at first 


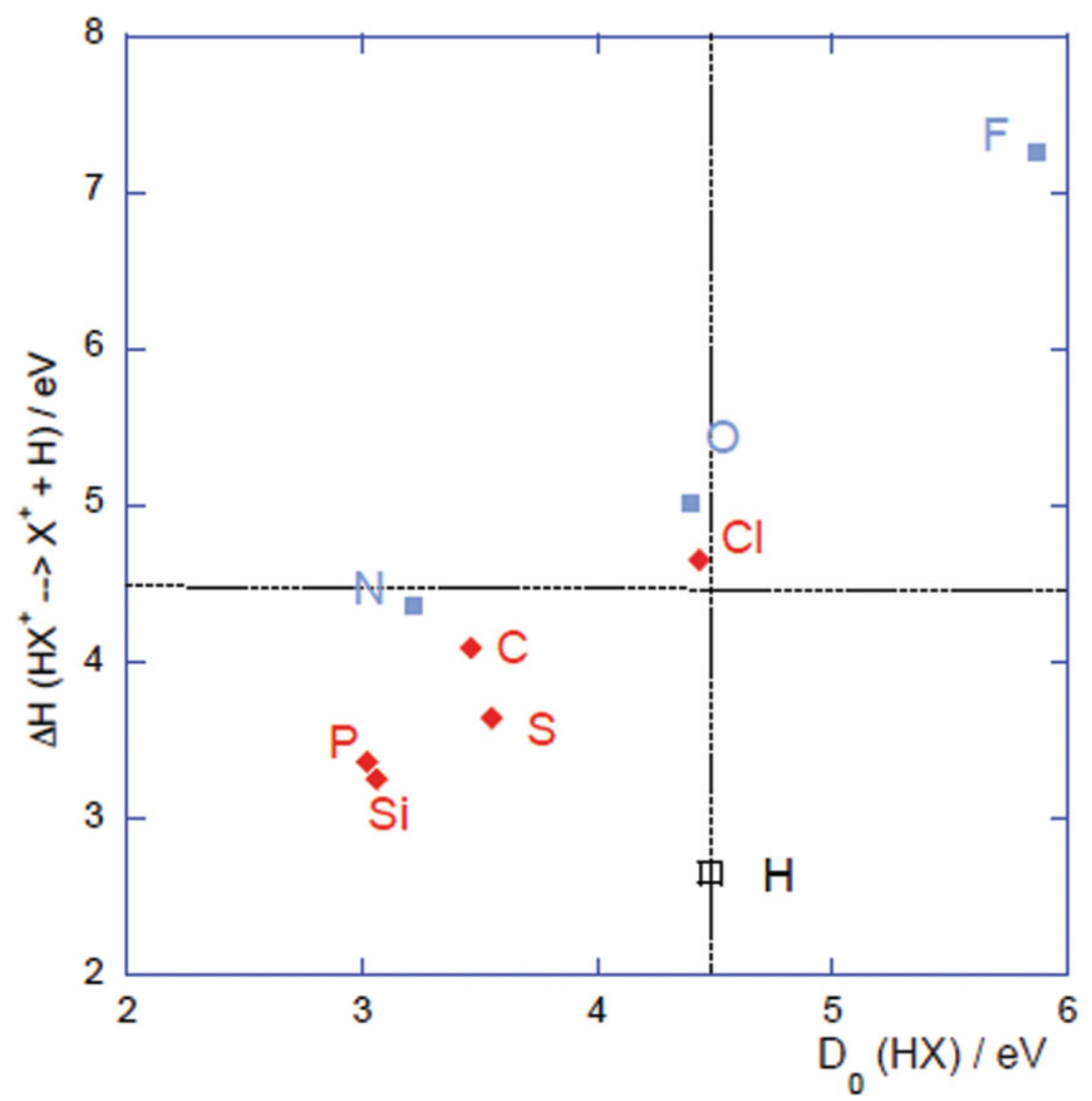

Figure 3. Energy released in the $\mathrm{XH}^{+} \rightarrow \mathrm{X}^{+}+\mathrm{H}$ reaction as a function of the dissociation energy of $\mathrm{XH}$ in $\mathrm{eV}$. Dissociation energy of $\mathrm{H}_{2}$ is reported as black dashed lines on both axis.

order from the following reactions:

$$
\begin{gathered}
\mathrm{H}_{2}+\zeta \rightarrow \mathrm{H}_{2}^{+}+e^{-} \\
\mathrm{H}_{2}^{+}+\mathrm{H}_{2} \rightarrow \mathrm{H}_{3}^{+}+e^{-} \\
\mathrm{H}_{3}^{+}+e^{-} \rightarrow \text { products }
\end{gathered}
$$

so that the steady state abundance of $\mathrm{H}_{3}^{+}$is given by the simple expression

$$
n\left(\mathrm{H}_{3}^{+}\right)=\zeta n\left(\mathrm{H}_{2}\right) / \alpha n(e),
$$

where $\alpha$ is the dissociative recombination rate of the $\mathrm{H}_{3}^{+}$molecular ion. If one assumes, as a first approximation, that the electrons are mainly coming from atomic carbon ions, the column density of $\mathrm{H}_{3}^{+}$is directly related to $\zeta$ through $\mathrm{N}\left(\mathrm{H}_{3}^{+}\right)=\zeta \mathrm{L} \mathrm{N}\left(\mathrm{H}_{2}\right) / \alpha \mathrm{N}\left(\mathrm{C}^{+}\right)$, where each factor, except $\zeta \mathrm{L}$, is known. As the dissociative recombination rate of $\mathrm{H}_{3}^{+}$is of the order of $2.6 \times 10^{-7}$, McCall et al. (2003) derived a value of $1.2 \times 10^{-15} \mathrm{~s}^{-1}$ towards the diffuse $\zeta$ Per line of sight. In a more detailed analysis of the complete molecular material in this line of sight, combined with several cloud components allowing to reproduce all observations within a factor of 3, Le Petit et al. (2004) derived a value of $\zeta$ of $2.5 \times 10^{-16}$ $\mathrm{s}^{-1}$ per $\mathrm{H}_{2}$, still higher than the standardly assumed ionization rate and above the lower limit derived by Liszt. The sample of diffuse locations, where $\mathrm{H}_{3}^{+}$has been detected, has been significantly extended recently by Indriolo \& McCall (2012). These authors were 

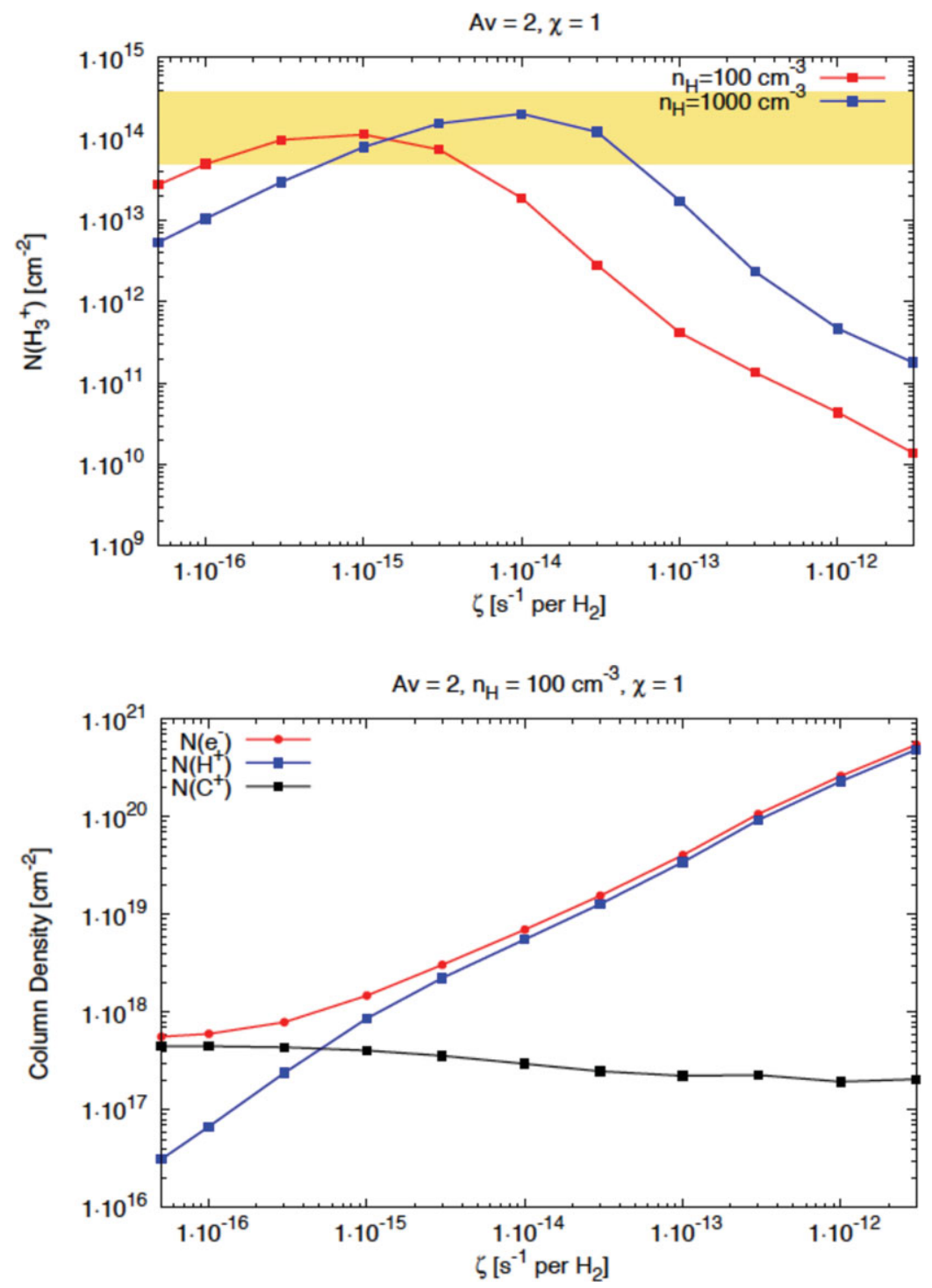

Figure 4. Upper panel: column densities of $\mathrm{H}_{3}^{+}$in function of $\zeta$ for 2 diffuse cloud models, $\mathrm{A}_{V}$ $=2, \mathrm{n}_{H}=100 \mathrm{~cm}^{-3}$ and $\mathrm{n}_{H}=1000 \mathrm{~cm}^{-3}$ and a standard ISRF. The yellow band reports the range of observations. Lower panel: column densities of electrons, carbon ions and protons for a cloud model $\mathrm{A}_{V}=2, \mathrm{n}_{H}=100 \mathrm{~cm}^{-3}$.

able to detect $\mathrm{H}_{3}^{+}$in more than 20 diffuse clouds, amongst which HD 183143 and HD 204827, well known sight lines where DIBs have been extensively studied and analyzed. They derive the cosmic ionization rate from expression 3.1, with values respectively of $(10.6 \pm 2.4)$ and $(9.32 \pm 2.4) \times 10^{-16} \mathrm{~s}^{-1}$. Such high ionization rates should have an impact on the possible carriers of DIBs.

\section{1. additional constraints}

The analysis described above relies however on several simplifying assumptions that we examine now. First, densities are assumed homogeneous so that column densities are directly obtained by multiplying the local densities by the linear scale of the cloud, 
second, destruction of $\mathrm{H}_{3}^{+}$by abundant neutrals is not taken into account, which is shown to be a good approximation in diffuse cloud conditions by Indriolo \& McCall (2012). However, the analysis performed by these authors is done by using the electron fraction as a free parameter, assuming implicitly that it is given by the amount of ionized carbon, although the range of electron fraction studied lies between $10^{-8}$ (dense cloud conditions) and $10^{-2}$. This upper limit is obviously raising some questions on the validity of this assumption as the elemental abundance of $\mathrm{C}^{+}$cannot exceed $\sim 2 \times 10^{-4}$.

We have run PDR models with the Meudon PDR code under diffuse cloud conditions where the density is assumed equal to $100 \mathrm{~cm}^{-3}$ and for a total visual extinction $\mathrm{A}_{V}=1$ for different values of the cosmic ionization rates. Upper panel of Fig. 4 displays the computed column density of $\mathrm{H}_{3}^{+}$in function of $\zeta$. The computed column density values are within the range of those detected in diffuse clouds for cosmic ionization rate values typically above $10^{-16}$. However, we see that, for cosmic ionization rates larger than $10^{-15}$, the computed column density of $\mathrm{H}_{3}^{+}$decreases. Indeed, at sufficiently high cosmic ionization rates, atomic and molecular hydrogen are significantly ionized, ionized carbon is no more a good proxy of the electronic fraction and the simple expression 3.1 fails, as also displayed in the lower panel of Fig. 4. For cosmic ionization rates larger than $\sim 10^{-14} \mathrm{~s}^{-1}$, protons become the main reservoir of charges and the simple analysis provided by expression 3.1 does not hold anymore. Similar conclusions are derived for isobaric models.

\section{4. $\mathrm{OH}^{+}, \mathrm{H}_{2} \mathrm{O}^{+}$}

These molecular ions have been very recently detected thanks to the Herschel satellite in front of high mass star forming regions in diffuse clouds (Gerin et al. 2012). We focus on the W51 diffuse line of sight where different molecules have been detected, including $\mathrm{H}, \mathrm{H}_{3}^{+}, \mathrm{C}^{+}, \mathrm{CH}, \mathrm{HF}, \mathrm{CO}, \mathrm{OH}, \mathrm{H}_{2} \mathrm{O}, \mathrm{OH}^{+}, \mathrm{H}_{2} \mathrm{O}^{+}$. Such a panel allows to test the cosmic ionization rate via two different probes, $\mathrm{H}_{3}^{+}$and $\mathrm{OH}^{+}, \mathrm{H}_{2} \mathrm{O}^{+}$as well as the ionization fraction via $\mathrm{C}^{+}$and molecular fraction via $\mathrm{H}, \mathrm{CH}$ and $\mathrm{CO}$. This gives a unique opportunity to constrain the physical conditions and corresponding models. As W51 is located at $2.5 \mathrm{kpc}$, different absorption clouds are lying on the line of sight at different velocities and we restrict the comparison to the $3-11 \mathrm{~km} / \mathrm{s}$ velocity range as reported in (Gerin et al. 2010; Sonnentrucker et al. 2010; Indriolo et al. 2012). We display in Table 2 different observational results from different telescopes (Arecibo, Herschel, SOFIA, UKIRT) and corresponding results for 2 diffuse cloud models.

We do not report the $\mathrm{CH}^{+}$and $\mathrm{SH}^{+}$observations reported in Godard et al. (2012) towards this line of sight, as these molecular ions require additional mechanisms which are not considered here.

We have run 2 different models corresponding to diffuse cloud conditions where the thermal balance is solved for a constant density model $\mathrm{n}_{H}=100 \mathrm{~cm}^{-3}$ and a constant pressure model with $\log _{10} \mathrm{p}=3.9$, where $\mathrm{p}$ is expressed in $\mathrm{cm}^{-3} \cdot \mathrm{K}$. These models are illuminated on one side and we stop the integration at a total visual extinction value $\mathrm{A}_{V}=2$, which corresponds approximately to the values derived from the deduced $\mathrm{H}$ and $\mathrm{H}_{2}$ column densities. Whereas an exact match between observations and models is not the purpose of the present paper, we find an encouraging agreement between observations and models when the cosmic ionization rate value is $10^{-15}$ for $\mathrm{H}_{3}^{+}, \mathrm{OH}^{+}$and $\mathrm{H}_{2} \mathrm{O}^{+}$. The column density of $\mathrm{CH}$ is below the observed value but we have dismissed possible shock or turbulent components which would account for $\mathrm{CH}^{+}$and $\mathrm{SH}^{+}$, and produce also by consequence additional $\mathrm{CH}$. The column density of $\mathrm{CO}$ is also well reproduced. However, the model overproduces the amounts of $\mathrm{OH}$ and then, the $\mathrm{OH} / \mathrm{H}_{2} \mathrm{O}$ ratio. This fact had 
Table 2. Molecular and Atomic column densities in the $3-11 \mathrm{~km} / \mathrm{s}$ component towards W51. Comparison with isobaric and isochoric models with $\zeta=10^{-15} \mathrm{~s}^{-1}, \chi=5$ for a total visual extinction $\mathrm{A}_{V}=2$.

\begin{tabular}{l|c|c|c|c|}
\hline Species & $\begin{array}{c}\text { Observations } \\
{\left[\mathrm{cm}^{-2}\right]}\end{array}$ & Ref & $\begin{array}{c}\text { isochoric model, } \mathbf{n}_{H}=\mathbf{1 0 0} \mathbf{~ c m - 3} \\
{\left[\mathrm{cm}^{-2}\right]}\end{array}$ & $\begin{array}{c}\text { isobaric model, } \mathbf{l o g}_{10} \mathbf{p}=\mathbf{3 . 9}{ }^{1} \\
{\left[\mathrm{~cm}^{-2}\right]}\end{array}$ \\
\hline $\mathrm{H}$ & $1.4810^{21}$ & 1 & $7.56 \times 10^{20}$ & $6.25 \times 10^{20}$ \\
$\mathrm{C}^{+}$ & $(4.0 \pm 0.4) \times 10^{17}$ & 2 & $4.05 \times 10^{17}$ & $3.32 \times 10^{17}$ \\
$\mathrm{CH}$ & $\left(3.7 \pm 0.2 \times 10^{13}\right)$ & 3 & $2.54 \times 10^{12}$ & $9.92 \times 10^{12}$ \\
$\mathrm{H}_{2}$ & $1.0 \times 10^{21}$ & 4 & $1.49 \times 10^{21}$ & $1.56 \times 10^{21}$ \\
$\mathrm{HF}$ & $\left(1.45 \pm 0.11 \times 10^{13}\right)$ & 5 & $3.54 \times 10^{13}$ & $3.59 \times 10^{13}$ \\
$\mathrm{H}_{3}^{+}$ & $(2.89 \pm 0.37) \times 10^{14}$ & 7 & $3.13 \times 10^{14}$ & $2.17 \times 10^{14}$ \\
$\mathrm{OH}^{+}$ & $(2.97 \pm 0.13) \times 10^{13}$ & 7 & $1.51 \times 10^{13}$ & $2.50 \times 10^{13}$ \\
$\mathrm{H}_{2} \mathrm{O}^{+}$ & $(6.09 \pm 0.96) \times 10^{12}$ & 7 & $1.15 \times 10^{13}$ & $7.69 \times 10^{12}$ \\
$\mathrm{CO}$ & $(2.81 \pm 0.21) \times 10^{15}$ & 7 & $2.76 \times 10^{15}$ & $2.87 \times 10^{15}$ \\
$\mathrm{OH}$ & $\left(6.4 \pm 0.9 \times 10^{13}\right)$ & 8 & $1.35 \times 10^{15}$ & $7.22 \times 10^{14}$ \\
$\mathrm{H}_{2} \mathrm{O}$ & $(6 \pm 3) \times 10^{13}$ & 9 & $1.37 \times 10^{14}$ & $1.59 \times 10^{13}$ \\
\hline $\mathrm{C}$ & & $8.62 \times 10^{16}$ & $1.19 \times 10^{18}$ \\
$\mathrm{O}$ & & $1.19 \times 10^{18}$ & $3.42 \times 10^{12}$ \\
$\mathrm{NH}$ & & $2.84 \times 10^{12}$ & $4.36 \times 10^{12}$ \\
$\mathrm{NH}_{2}$ & & $7.54 \times 10^{11}$ & $4.82 \times 10^{12}$ \\
$\mathrm{H}_{3} \mathrm{O}^{+}$ & & $1.10 \times 10^{13}$ & \\
\hline
\end{tabular}

Notes: ${ }^{1} \mathrm{p}$ in $\mathrm{cm}^{-3} \cdot \mathrm{K}$

References: (1) Koo (1997) for an assumed spin temperature of 100K, (2) Gerin et al. (2012) (3) Gerin et al. (2010), (4) derived from $\mathrm{CH}$ through $\mathrm{N}\left(\mathrm{H}_{2}\right)=\mathrm{N}(\mathrm{CH}) / 3.5 \times 10^{-8}$ (Sheffer et al. 2008), (5) Sonnentrucker et al. $(2010),(6)$ derived from HF, (7) Indriolo et al. (2012), (8) Wiesemeyer et al. (2012); Neufeld et al. (2002), (9) Flagey et al. (2013)

already been raised by Le Petit et al. (2004) in their study of the $\zeta$ Per line of sight, as increasing the cosmic ionization rate also favors production of $\mathrm{OH}$, whose starting step relies on the production of $\mathrm{O}^{+}$through the $\mathrm{H}^{+}+\mathrm{O}$ charge transfer reaction. We additionally display the column density of other species which may be searched, such as atomic carbon and oxygen, $\mathrm{H}_{3} \mathrm{O}^{+}$and nitrogen hydrides. It is remarkable that the computed column density of atomic oxygen is the same in both models, which reflects that atomic oxygen is the reservoir of oxygen in these diffuse regions and depend only on the total column density of protons. The corresponding observations would help to constrain the amount of available oxygen which will impact $\mathrm{OH}, \mathrm{H}_{2} \mathrm{O}$ formation.

We display in Figure 5 column densities of $\mathrm{OH}^{+}, \mathrm{H}_{2} \mathrm{O}^{+}$and $\mathrm{H}_{3}^{+}$as a function of $\mathrm{A}_{V}$ obtained in the previously referred isobaric model. We confirm that these ions are formed close to the edge of the cloud, as pointed out in Neufeld (2010), when the molecular fraction is low, whereas $\mathrm{H}_{3}^{+}$is formed at higher extinction in a fully molecular region. Two independent recent studies of comic ray propagation taking into account magnetic effects (Rimmer et al. 2012; Padovani \& Galli 2013), suggest that the cosmic ionization rate factor could vary from the edge to the interior of interstellar clouds. Such an effect has been tested against the presence of carbon chain in the Horsehead nebula (Rimmer et al. 2012). It could also apply to the simultaneous detection of $\mathrm{OH}^{+}, \mathrm{H}_{2} \mathrm{O}^{+}, \mathrm{H}_{3}^{+}$which probe different spatial environments in a single diffuse cloud so that large cosmic ionization rates could be used to interpret $\mathrm{OH}^{+}$and $\mathrm{H}_{2} \mathrm{O}^{+}$whereas somewhat lower cosmic ionization rates would apply to $\mathrm{H}_{3}^{+}$.

The high cosmic ionization rate derived from the simultaneous detection of $\mathrm{OH}^{+}$and $\mathrm{H}_{2} \mathrm{O}^{+}$has motivated high energy physicists to predict fluxes of nuclear $\gamma$ - ray lines from the inner Galaxy which could be measurable by new-generation $\gamma$ - ray telescopes based on available technology (Benhabiles-Mezhoud et al. 2013). 


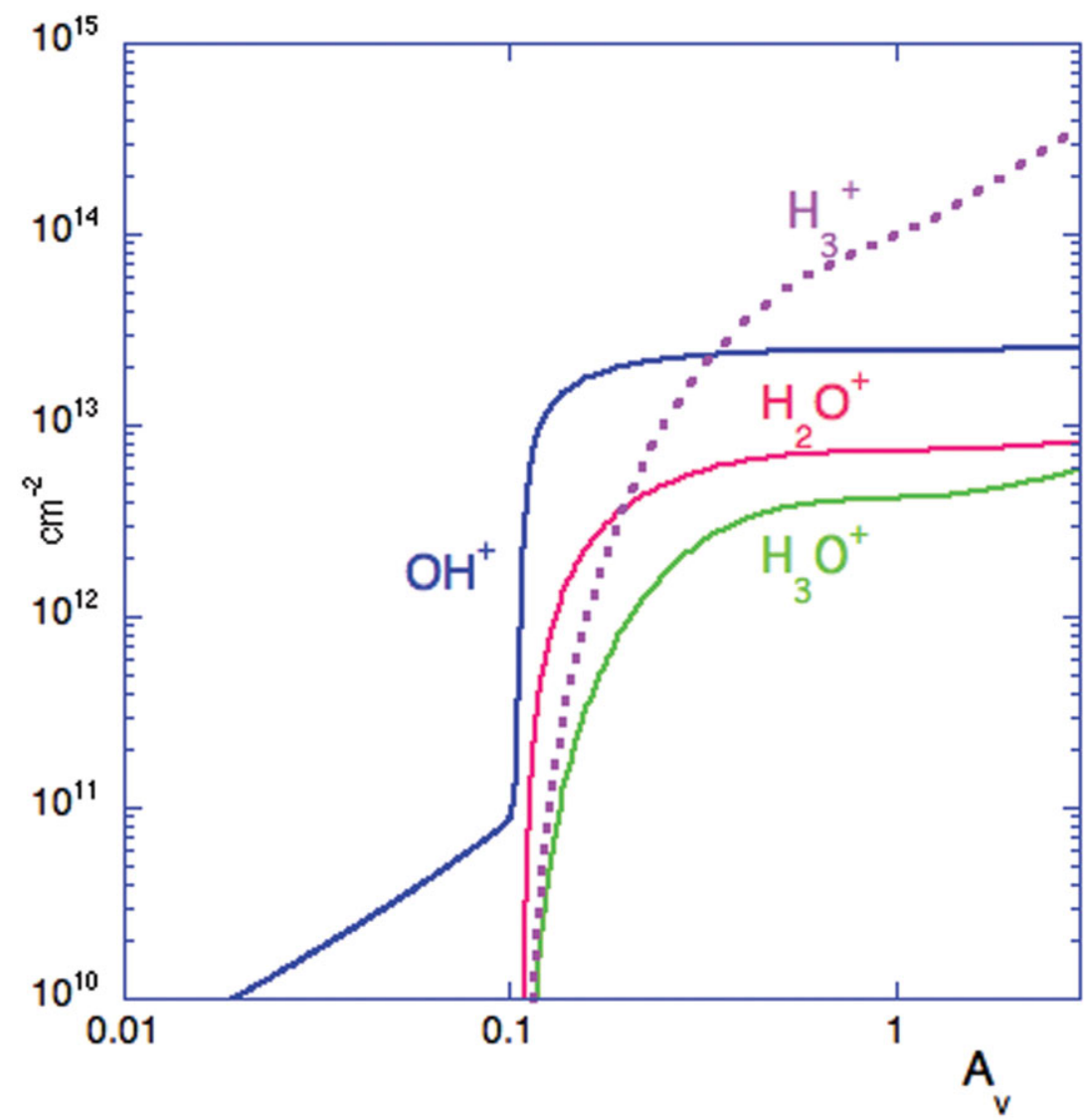

Figure 5. Computed column densities as a unction of visual extinction $A_{V}$ for an isobaric model with $\log (\mathrm{p})=3.9 \mathrm{~cm}^{-3} \cdot \mathrm{K}, \zeta=10^{-15} \mathrm{~s}^{-1}$ and a scaling factor of 5 for the incident radiation field in Mathis units.

\section{Conclusions}

Diffuse cloud models involve a variety of physico-chemical processes where matterradiation effects play a major role. A general consensus on the basic gas phase physical mechanisms has been reached between different groups involved in astrophysical modeling of these regions (Röllig et al. 2007). The role of dust phase interaction is still debated and involves many unknown factors including the nature of dust particles (silicate, graphite/amorphous carbon), their size distribution which have a direct impact on the temperature via photoelectric effect and also on the chemistry. $\mathrm{H}_{2}$ is well known to be formed on surfaces and the corresponding formation rate still deserves further studies although the overall formation rate looks rather well constrained, thanks in great part to the simultaneous detection of atomic and molecular hydrogen with former UV satellites. A new important result has been reached from recent far infrared Herschel results, confirming the large value of cosmic ionization rate first deduced from $\mathrm{H}_{3}^{+}$. The impact on those uncertainties on DIBs is difficult to assess but they certainly have to be taken into account in any attempt to identify the carriers of these yet unidentified features. 


\section{Acknowledgements}

We acknowledge support from ANR programme blanc SUMOSTAI and Programme National PCMI (Physico-Chimie du Milieu Interstellaire).

\section{References}

Abgrall, H., Le Bourlot, J., Pineau Des Forets, G., et al. 1992, A\&A, 253, 525

Benhabiles-Mezhoud, H., Kiener, J., Tatischeff, V., \& Strong, A. W. 2013, ApJ, 763, 98

De Luca, M., Gupta, H., Neufeld, D., et al. 2012, ApJL, 751, L37

Draine, B. T. 1978, ApJS, 36, 595

Flagey, N., Goldsmith, P. F., Lis, D. C., et al. 2013, ApJ, 762, 11

Gerin, M., de Luca, M., Goicoechea, J. R., et al. 2010, A\&A, 521, L16

Gerin, M., Levrier, F., Falgarone, E., et al. 2012, Royal Society of London Philosophical Transactions Series A, 370, 5174

Godard, B., Falgarone, E., Gerin, M., et al. 2012, A\&A, 540, A87

Goicoechea, J. R. \& Le Bourlot, J. 2007, A\&A, 467, 1

Gonzalez Garcia, M., Le Bourlot, J., Le Petit, F., \& Roueff, E. 2008, A\&A, 485, 127

Indriolo, N. \& McCall, B. J. 2012, ApJ, 745, 91

Indriolo, N., Neufeld, D. A., Gerin, M., et al. 2012, ApJ, 758, 83

Jenkins, E. B. \& Tripp, T. M. 2011, ApJ, 734, 65

Koo, B.-C. 1997, ApJS, 108, 489

Le Bourlot, J., Le Petit, F., Pinto, C., Roueff, E., \& Roy, F. 2012, A\&A, 541, A76

Le Petit, F., Barzel, B., Biham, O., Roueff, E., \& Le Bourlot, J. 2009, A\&A, 505, 1153

Le Petit, F., Nehmé, C., Le Bourlot, J., \& Roueff, E. 2006, ApJS, 164, 506

Le Petit, F., Roueff, E., \& Herbst, E. 2004, A\&A, 417, 993

Le Petit, F., Roueff, E., \& Le Bourlot, J. 2002, A\&A, 390, 369

Lique, F., Li, G., Werner, H.-J., \& Alexander, M. H. 2011, J. Chem. Phys., 134, 231101

Liszt, H. 2003, A\&A, 398, 621

McCall, B. J., Hinkle, K. H., Geballe, T. R., et al. 2002, ApJ, 567, 391

McCall, B. J., Huneycutt, A. J., Saykally, R. J., et al. 2003, Nature, 422, 500

Neufeld, D. A. 2010, ApJ, 708, 635

Neufeld, D. A., Goicoechea, J. R., Sonnentrucker, P., et al. 2010, A\&A, 521, L10

Neufeld, D. A., Kaufman, M. J., Goldsmith, P. F., Hollenbach, D. J., \& Plume, R. 2002, ApJ, 580,278

Neufeld, D. A. \& Wolfire, M. G. 2009, ApJ, 706, 1594

Padovani, M. \& Galli, D. 2013, Astrophysics and Space Science Proceedings, 34, 61

Rachford, B. L., Snow, T. P., Destree, J. D., et al. 2009, ApJS, 180, 125

Rimmer, P. B., Herbst, E., Morata, O., \& Roueff, E. 2012, A\&A, 537, A7

Röllig, M., Abel, N. P., Bell, T., et al. 2007, A\&A, 467, 187

Sheffer, Y., Rogers, M., Federman, S. R., et al. 2008, ApJ, 687, 1075

Snow, T. P. \& McCall, B. J. 2006, ARAA, 44, 367

Sonnentrucker, P., Neufeld, D. A., Phillips, T. G., et al. 2010, A\&A, 521, L12

van Dishoeck, E. F. \& Black, J. H. 1986, ApJS, 62, 109

Wiesemeyer, H., Güsten, R., Heyminck, S., et al. 2012, A\&A, 542, L7 